\section{Intersections}

Canadian Journal of Music

Revue canadienne de musique
Intersections CANADIAN JOURAL OF MUSIO

\title{
Reflets de la colonialité dans la scène des musiques nouvelles
}

\section{Gabriel Dharmoo}

Volume 39, numéro 1, 2019

Decolonizing Music Pedagogies

URI : https://id.erudit.org/iderudit/1075345ar

DOI : https://doi.org/10.7202/1075345ar

Aller au sommaire du numéro

Éditeur(s)

Canadian University Music Society / Société de musique des universités canadiennes

ISSN

1918-512X (numérique)

Découvrir la revue

Citer cet article

Dharmoo, G. (2019). Reflets de la colonialité dans la scène des musiques nouvelles. Intersections, 39(1), 105-121. https://doi.org/10.7202/1075345ar

\section{Résumé de l'article}

Certains membres de la scène des musiques nouvelles souhaitent en décentrer ses racines eurocentriques et en critiquer ses tendances colonialistes. Avant même de discuter des stratégies qui pourraient constituer un cadre décolonisateur, il est utile d'identifier comment la colonialité se reflète dans cette scène. L'auteur, lui-même membre actif de celle-ci, partage des pistes de réflexion portant sur l'homogénéité culturelle du milieu, les questions d'accès, l'héritage de la musique classique, le concept de l'excellence européenne, la présomption d'universalité, la coexistence de statuts de légitimité et de marginalité, la relation ambigüe avec l'appropriation culturelle et les fondements de l'attribution du mérite.
C Canadian University Music Society / Société de musique des universités canadiennes, 2021
Ce document est protégé par la loi sur le droit d'auteur. L'utilisation des services d'Érudit (y compris la reproduction) est assujettie à sa politique d'utilisation que vous pouvez consulter en ligne.

https://apropos.erudit.org/fr/usagers/politique-dutilisation/ 


\title{
REFLETS DE LA COLONIALITÉ DANS LA SCÈNE DES MUSIQUES NOUVELLES
}

\author{
Gabriel Dharmoo
}

La scène de la musique d'art occidentale contemporaine (ci-après appelée «scène des musiques nouvelles») fait l'objet d'appels de plus en plus nombreux à décentrer ses racines eurocentriques et à critiquer ses origines et tendances colonialistes, impérialistes, capitalistes, institutionnelles, racistes, hétéronormatives ou patriarcales, entre autres ${ }^{1}$. En convergence avec les sujets couramment discutés depuis plusieurs générations ${ }^{2}$, on remarque une considération grandissante envers les problématiques reliées à la «décolonisation» du milieu. Bien que ces enjeux soient davantage discutés dans le milieu du théâtre et de la danse, la scène des musiques nouvelles réfléchit de plus en plus à sa relation avec les communautés autochtones et les artistes issus des Premières Nations, la représentation de la diversité culturelle ainsi que la collaboration interculturelle. Ces sujets sont abordés lors d'événements organisés pour susciter la discussion 3 , mais aussi lors de conversations informelles entre collègues. Il faut faire preuve de discernement avant de tous les assimiler à un processus dit de "décolonisation». Tuck et Yang (2012) dénoncent l'attribution du terme à des modèles de justice sociale antérieurs à la popularisation de ce cadre d'analyse, un cadre qui, soutiennent-ils, doit prioritairement, voire exclusivement, traiter des problématiques reliées au colonialisme réel et persistant à l'égard des Premières Nations. Bien que les enjeux mis en lumières par Tuck et Yang m’aient porté à réfléchir profondément sur les manières d'exercer et d'exprimer ma solidarité à cette cause, c'est la définition plus large de Levitz (2017) qui me servira de modèle pour cet article. Dans ses recommandations pour la décolonisation

1 Pour leurs suggestions, références et relectures, je tiens à remercier Sandeep Bhagwati, Noah Drew, Erin Gee, Symon Henry, David Howes, Kama La Mackerel et Catherine Lefrançois, en plus du comité d'édition d'Intersections.

2 Mentionnons la parité des genres dans la programmation de concerts et de festivals, la démocratisation des événements, le renouvèlement des protocoles de concert, la diversification des publics, la pertinence du modèle de commande d'œuvres, la primauté de la partition versus la comprovisation ou l'improvisation, les systèmes de notation alternatifs, les processus de création collaborative ou interdisciplinaire, ou encore l'impact des arts communautaires.

3 Quelques exemples de conférences qui abordaient ces sujets : "Compositeurs : une espèce en voie de disparition?» (Montréal/Nouvelles Musique 2019), "Forum Entr’arts» (Réseau canadien des musiques nouvelles 2018), «Horizons musicaux contemporains» (Montréal/Nouvelles Musique 2017), «Démocratisation de la performance» (Ligue canadienne des compositeurs 2015). 
de la SAM (Society of American Music), elle décrit un "projet collectif qui consiste à théoriser et à clarifier la colonialité du pouvoir, à prendre en compte ses conséquences spatiales et temporelles, puis à adopter une grande variété de mesures pour y remédier» (Levitz 2017, 2) ${ }^{4}$. Suivant la logique de cette définition tripartite, il est utile d'identifier comment la colonialité du pouvoir se reflète dans la scène des musiques nouvelles avant même de discuter des mesures et des stratégies qui pourraient en constituer un cadre décolonisateur.

Cet article réflexif s'appuie sur mes propres opinions, observations et expériences au sein de la scène des musiques nouvelles, où j'opère professionnellement depuis plus de quinze ans à titre de compositeur, improvisateur et interprète vocal. Selon différents contextes, j'occupe alternativement d'autres rôles: chercheur, commissaire, administrateur, organisateur, médiateur culturel ou mentor. Dans ces différents rôles et projets, j'interagis avec une grande variété d'individus impliqués de près ou de loin dans cette scène : compositeurs et compositrices, improvisateurs et improvisatrices, artistes sonores, musiciens et musiciennes au sein d'ensembles de musique nouvelle ou classique, directions artistiques, commissaires de festivals, ainsi que plusieurs individus ayant des rôles pédagogiques, artistiques ou administratifs dans des institutions d'études supérieures (Conservatoires ou départements et facultés de musique universitaires), des institutions artistiques (Groupe Le Vivier et ses organismes membres, Société de musique contemporaine du Québec), des associations (Conseil québécois de la musique, Centre de musique canadienne, Ligue canadienne des compositeurs, SOCAN), des réseaux (Réseau canadien des musiques nouvelles), des subventionnaires (Conseil des arts du Canada, Conseil des arts et des lettres du Québec) et plusieurs autres organismes. Mes réseaux me portent à travailler au Canada comme à l'international. J'ai donc une connaissance particulièrement approfondie du milieu par l'entremise de mes activités professionnelles, mais d'autres facteurs contribuent à ma perspective critique du milieu et à mon désir de réfléchir à son avenir décolonial : ma positionalité en tant que personne de couleur et mon identité biculturelle (mon père étant indo-caribéen et ma mère québécoise), mon apprentissage et mes incursions artistiques complémentaires dans d'autres traditions musicales suite à une formation de base en musique classique occidentale, ma prédilection pour la collaboration interculturelle, puis finalement, la diversification graduelle et volontaire de mes publics et de mes partenaires.

La majeure partie de cet article se penche sur l'identification et la description de différents reflets de la colonialité dans la scène : l'homogénéité culturelle du milieu, les questions d'accès, l'héritage de la musique classique, le concept de l'excellence européenne, la présomption d'universalité, la coexistence de statuts de légitimité et de marginalité, la relation ambigüe avec l'appropriation culturelle et les fondements de l'attribution du mérite. J'espère que ces constats, ces idées et ces pistes de réflexion puissent expliquer, contextualiser, inspirer et aider à renforcer l'application de diverses stratégies et politiques

4 «a collective project that involves theorizing about and clarifying coloniality of power, considering its spatial and temporal consequences, and taking a wide range of actions to counter it». 
visant la décolonisation du milieu, ou mener à des recherches académiques supplémentaires.

Afin d'identifier certaines pistes de solutions concrètes, j'ouvrirai également sur les stratégies dont le milieu des musiques nouvelles pourrait se doter. Le récent rapport d'enquête L'orchestre retentit (2019), co-écrit par Attariwala et Peerbaye et commandé par Orchestres Canada, analyse de près certains des aspects problématiques des relations entre les orchestres canadiens, les peuples autochtones et les personnes de couleur. Il s'agit d'une base pour réfléchir et remédier à la colonialité en musique, cependant, le milieu orchestral gravite autour d'un répertoire du passé, tandis que la scène des musiques nouvelles s'auto-identifie comme un art de notre temps, tourné vers l'avenir. Les enjeux et les stratégies décoloniales potentielles sont de nature différente. Considérant mon propre profil d'artiste, le court sommaire des stratégies créatives et professionnelles que je propose est relié à l'idée de décolonisation par l'art exprimée par Sioui-Durand (2018).

\section{HoMogénéité CULTURELLE ET ASSIMILATION DE LA DIFFÉRENCE}

La scène de la musique nouvelle au Québec est culturellement homogène et majoritairement blanche. Les individus racisés et les autochtones qui s'identifient comme appartenant à la scène ont tendance à employer un discours artistique qui est au service, qui répond aux attentes ou qui est compatible avec la perspective ou les intérêts de la culture dominante. La différence culturelle a tendance à être assimilée, adaptée ou reformatée pour mieux appartenir aux définitions ou aux délimitations du genre. Ce processus peut mener à des résultats artistiques de grande valeur et indique si le rapport d'un artiste à une tradition donnée vise la préservation, la reproduction, la transgression, l'expérimentation ou une autre stratégie créative. Cependant, les individus issus de la culture dominante œuvrant au sein de la scène ont rarement les outils pour mesurer la part de tradition et d'innovation dans des musiques provenant ou inspirées d'une autre culture; j'y reviendrai. Certaines de mes compositions inspirées par des éléments de la musique carnatique indienne ont été bien reçues par les membres du milieu, mais derrière cette appréciation se cache souvent un dédain colonialiste envers d'autres tentatives de métissage qui sont jugées trop près de la «musique du monde».

Devant le constat de cette homogénéité culturelle, on soulève souvent le fait que la scène compte plusieurs individus ayant un bagage culturel «asiatique». Dans une section du rapport d'enquête L'orchestre retentit portant sur l'élitisme culturel et l'Extrême-Orient, Attariwala réfère à Yoshihara, qui décrit les "processus grâce auxquels les Asiatiques ont adopté la musique classique dans leurs cultures», des processus "qui sont tous étroitement liés au besoin de se moderniser» (Attariwala, Peerbaye 2019, 46). Dans une récente correspondance électronique, Attariwala me réitère comment Yoshihara, selon elle, «indique très clairement que la participation de la Chine, du Japon et de la Corée aux arts occidentaux a été (après la Seconde Guerre mondiale et la 
Grande révolution culturelle prolétarienne) calculée pour gagner du capital culturel en imitant la culture expressive de l'élite occidentale; en tandem avec les tentatives de ces pays de prendre pied dans l'économie mondiale, qui était, à l'époque, dominée par l'Occident » (Attariwala, correspondance avec l'auteur, 2019)5. Attariwala souligne que "pour l'Asie du sud et l'Afrique, des différences distinctes existent par rapport à la situation en Asie de l'est, différences reliées à leur colonisation et aux réclamations postcoloniales et résurgences culturelles ultérieures» (Attariwala, corresp. avec l'auteur, 2019) ${ }^{6}$. En fait, «le Japon, la Corée et la Chine (exception faite de petits territoires) ne faisaient pas partie des empires coloniaux de l'Europe et leur estimation de la musique occidentale n’est pas tachée par le colonialisme» (Attariwala et Peerbaye 2019, 45). Bien que ces groupes culturels soient représentés sur la scène des salles de concert, il en demeure que «les parents et les élèves, qui sont prêts à payer pour des leçons de musique, ne sont pas habitués à payer pour assister à un concert en raison de leur culture» (Attariwala et Peerbaye 2019, 47). La diversité acquise par ces processus de gain en capital culturel ne se reflète donc pas de façon significative dans la démographie du public de musique orchestrale.

Il faut aussi reconnaitre comment l'effacement des figures Noires dans l'histoire de la musique classique envoie un message de non-inclusion qui persiste à ce jour. Green (2018) mentionne comment Ignatius Sancho, Chevalier de Saint-Georges, Blind Tom, Florence Price, Margaret Bonds, William Grant Still, et plusieurs autres compositrices et compositeurs sont écartés des cursus universitaires, à l'exception de cours spécialisés. «Pourquoi, par exemple, ne pas inclure le chevalier de Saint-Georges dans un cours général d'histoire de la musique?» (Green 2018)7. Cette omission, parmi d'autres, des institutions universitaires «envoie un message aux compositrices et compositeurs Noirs [qu'ils] ne sont pas les bienvenus, quel que soit le succès [qu'ils] remportent. La scène des musiques nouvelles a très peu contribué à changer l'optique attendue de la musique classique, ce qui explique pourquoi le problème d'identité de la scène est ce qu'il est aujourd'hui» (Green 2018) ${ }^{8}$.

Des personnes d'origines culturelles différentes sont parfois invitées à participer à la scène, le temps d'un projet, d'un événement, d'une conférence, mais le geste est souvent considéré comme un privilège à accueillir avec reconnaissance. En réalité, c'est souvent l'artiste invité qui concède la plus grande part de compromis ou qui fait preuve d'adaptation, que ce soit par rapport au contexte

5 «She makes it very clear that Chinese, Japanese, Korean involvement in Western arts was (post WWII and post-Cultural Revolution) were calculated moves to gain cultural capital by imitating elite Western expressive culture; in tandem with these countries' attempts to gain footholds in the global economy, which was - at the time - dominated by the West».

6 « [... ] there are distinct differences between East Asian and South Asian/African expressive culture ideals that are related to the colonization (and subsequent postcolonial reclamations and cultural resurgences) of the latter $\%$.

7 "Why not, for example, include Chevalier de Saint-Georges in a general music history class? »

8 "Yet his and other black composers' non-existence in academic institutions tells black composers that we are not wanted, no matter how much success we gain. New music has done very little to change the expected optics of classical music, which is why new music's identity problem is what it is today". 
de présentation de son travail, au matériau musical ou aux considérations artistiques mêmes. Du côté des organismes hôtes, des retombées positives se feront sentir. Leur réputation s'enrichira tant aux yeux du public quaux yeux des subventionneurs qui encouragent les organismes à se réinventer dans cette direction. Une fois la collaboration terminée, qui bénéficie véritablement de ces projets? L'exercice de décentralisation et d'ouverture à la différence ne devrait-elle pas entraîner une part de sacrifice et de concessions chez l'organisme en position de pouvoir?

Si un projet de musique interculturelle impliquant une participation autochtone vise à éduquer le public (majoritairement blanc) sur les atrocités de la colonisation, tentant ainsi de contribuer aux processus de réparation et de réconciliation, Robinson (2012) analyse comment l'expérience affective et sensorielle du public envers la musique et le concept artistique peut se confondre avec un sentiment de finalité, de réelle réconciliation:

La performance d'une musique interculturelle peut être vécue comme une réconciliation symbolique lorsque le public a le sentiment que quelque chose a réellement été accompli. En effet, même si le 'pouvoir de la musique' peut avoir de nombreux avantages, il peut tout aussi bien convaincre son public que [ ... ] l'expérience d'une telle performance est en fait la réconciliation en soi (Robinson 2012, 123) 9 .

De telles collaborations interculturelles peuvent «rendre visible une forme de réconciliation, mais celle-ci est dépourvue d'outils pouvant mener à l'action» (Robinson 2012, 120) ${ }^{10}$.

D'autres fois, de tels projets sont initiés par des autochtones ou des minorités culturelles, mais cela n'empêche pas que la négociation interculturelle peut susciter simultanément des éloges et des critiques. En réaction à la tournée du Powwow Symphony de Brent Michael Davids, Robinson recueille les propos de Waziataywin pour qui «la tournée était trop axée sur l'apparence d'une réconciliation sans la substance de la réparation» (Robinson 2012, 122) ${ }^{11}$. Si la solidarité interculturelle n'est qu'une façade, un simple geste symbolique qui n'a que peu de répercussions réelles, est-ce là une des limites de l'œuvre d'art? Les solutions tangibles reposent-elles dans tout ce qui est périphérique à l'œuvre, par exemple son contexte de présentation ou la médiation culturelle? Le simple fait de séparer l'œuvre de son contexte est-il une limite dont le milieu peut se défaire?

9 «The feeling that something has been achieved positions such intercultural music performance as symbolic reconciliation and something more. Indeed, as much as the very 'power of music' might have a range of benefits, it might just as well convince its audiences, that $[\ldots]$ the experiencing of such performance is the doing of reconciliation itself".

10 " a form of reconciliation made visible, but lacking adequate provision for action ".

11 "The tour was too much about the appearance of reconciliation without the substance of redress". 


\section{QUESTIONS D'ACCÈS}

En ce qui concerne les interprètes, la réussite ou la simple inclusion à la scène des musiques nouvelles est fondée sur le mérite. Ainsi, l'acquisition des compétences nécessaires pour travailler au sein de cette scène exige une formation approfondie qui passe presque nécessairement par l'apprentissage musical en privé ou dans un programme scolaire spécialisé dès l'enfance, puis d'une formation dans un conservatoire ou une université en musique. Ce parcours correspond généralement à celui de la classe moyenne et riche. Les privilèges socioéconomiques et raciaux comme la richesse, la blanchitude, le niveau d'éducation des parents et l'appartenance à une culture dominante sont manifestement évidents, mais ils auraient intérêt à être davantage discutés et problématisés afin de les lier directement aux questions d'accès et de mérite; toutes ces années de formation musicale visent la progression vers une virtuosité autrement inatteignable.

Pour les compositrices et compositeurs et autres artistes sonores, lorsqu'il est question d'accès, on n'observe pas d'obstructions agressives sur la base du genre, de l'ethnicité ou de l'orientation sexuelle. Notre sous-culture issue de l'avant-garde privilégie les voix artistiques uniques, ce qui peut être compatible avec la célébration de la différence ou de l'altérité stylistique. En apparence, les portes sont grandes ouvertes et les politiques des subventionneurs en matière de diversité contribuent à l'image d'une scène artistique progressiste et inclusive. Pourtant, tel que démontré par Ariane Couture (2019), un déséquilibre subsiste quant au genre des compositeurs dont les œuvres sont programmées par les principaux organismes de musique nouvelle à Montréal. L'absence de recherche approfondie ou de statistiques sur les compositeurs ${ }^{12}$ non-blancs indique que nous en sommes encore au stade des cas d'exception. N'oublions pas que des problèmes structurels font en sorte qu'il est difficile pour les compositeurs non-occidentaux ou immigrants d'accéder au même type d'éducation, de financement et de possibilités. Considérant le profil type du compositeur issu d'une éducation en musique et de l'apprentissage d'un instrument, il convient de faire un lien avec les pistes offertes dans L'orchestre retentit afin d'améliorer l'accès aux ressources d'apprentissage. Le rapport de consultation souligne également le besoin de soutien supplémentaire continu et de mentorat pour contrer, par exemple, les conditions économiques, culturelles et familiales qui freinent ce type de choix de carrière.

Ces compositrices et compositeurs sont souvent privés du réseautage déterminant pendant les années de formation. Si je pense à l'ensemble des opportunités qui m’ont été offertes dans ma carrière, je peux souvent en retracer l'origine à un lien personnel développé pendant mes études. Un compositeur nouvellement immigré et formé à l'étranger (surtout à l'extérieur des pays dits occidentaux) réussit plus difficilement à tisser ces liens et à en subir des retombées tangibles. Pour de multiples raisons très compréhensibles, les directions artistiques, ensembles ou commissaires peuvent avoir tendance à privilégier

12 Dans cette section, le mot «compositeur» est utilisé de manière épicène et désigne tant les compositrices que les compositeurs. 
des relations professionnelles déjà garantes de succès. La collaboration interculturelle ou avec des personnes issues de l'immigration amène des défis et des risques que certains accueillent avec enthousiasme, d'autres avec un pragmatisme contraignant, et ce, peu importe s'ils œuvrent dans un langage traditionnellement différent, ou compatible aux musiques nouvelles.

Si les portes sont ouvertes, je me questionne : qui veut vraiment y entrer? Les musiciennes et musiciens qui ont des approches novatrices ou expérimentales et qui souscrivent à une autre tradition ont-ils l'impression que la scène des musiques nouvelles serait prête à recevoir leur travail ? Sont-ils artistiquement plus épanouis, mieux compris et mieux soutenus ailleurs? La scène compteelle assez de modèles issus de la diversité culturelle pour créer un sentiment d'inspiration pour les générations plus jeunes?

\section{L'HÉRITAGE ET L'ENCHEVÊTREMENT AVEC LA MUSIQUE CLASSIQUE OCCIDENTALE}

Je me permets une analogie boiteuse : la musique classique occidentale agit à titre de grands-parents des musiques nouvelles. Si les musiques nouvelles accueillent chaleureusement la bienveillance et les encouragements de la musique classique, elles la jugent parfois trop démodée, déconnectée ou répréhensible. L'influence de cet héritage est cependant évidente chez les musiciennes et musiciens, qui maintiennent souvent un rapport intime avec la musique classique par l'entremise de différents postes orchestraux ou d'enseignement, des contrats réguliers ou ponctuels. En ce qui concerne les créatrices et créateurs en composition et an art sonore, la situation est plus complexe. Un certain nombre prétendra s'être détaché de leurs racines classiques occidentales. D'autres, qui proviennent d'une formation non-classique (autodidactes, jazz, musique populaire) prétendront n'avoir que peu en commun avec cette musique historique. Mais il est indéniable que la scène a hérité des modèles et des outils de cette musique eurologique ${ }^{13}$ : le financement par subvention, la primauté des partitions et de la notation, le paradigme de la commande d'œuvre, le protocole et l'expérience de concert, les rôles et la hiérarchie, la lutherie des instruments qui sont appris, joués et pour lesquels on compose.

$\mathrm{Au}$ Québec, la plupart des organismes de musique nouvelle coexistent et interagissent avec les organismes de musique classique occidentale, sous le parapluie d'organismes comme le Conseil Québécois de la Musique ou le média en ligne Ludwig Van Montréal. Les organismes de la scène souscrivent volontairement à cette grande famille de la musique classique occidentale par le biais d'adhésions payantes - ce qui dénote une association claire, consciente et consensuelle.

Bien que de nombreux sous-genres de musique nouvelle aient évolué loin de la musique classique - pensons à la musique improvisée/actuelle, ou encore aux arts sonores et médiatiques - ces avenues alternatives n'évoluent pas

13 «Désigne tout musicking - quelle que soit son origine géographique - qui a) se situe à l'intérieur et b) présente plusieurs caractéristiques saillantes de la tradition musicale occidentale» (Sandeep Bhagwati,. «Glossaire raisonné», Circuit - Musiques contemporaines 28 (1), 2018, p.15). 
nécessairement vers un avenir décolonial ou loin d'autres préjugées problématiques. Par exemple, le sexisme et le manque de diversité sexuelle dans les milieux reliés aux technologies musicales, à l'improvisation libre et au jazz expérimental sont souvent critiqués ${ }^{14}$. La reconnaissance de différents systèmes d'oppression liés à l'identité (culture, ethnicité, spiritualité, genre, sexualité, classe, éducation) permet une décentralisation intersectionnelle du pouvoir.

\section{L'EXCELLENCE EUROPÉENNE}

«La terminologie et les propos qui caractérisent la musique classique occidentale comme étant particulièrement 'excellente' et 'universelle' proviennent de l'empire, du colonialisme et du capitalisme» (Attariwala et Peerbaye 2019, 39) ${ }^{15}$. Qu'elle le veuille ou non, la musique classique occidentale est une célébration de l'excellence historique européenne (et blanche). Dans le contexte actuel, on conteste rarement le fait qu'il s'agit là d'un marqueur de la prédominance des Blancs. Examinons de près les slogans respectifs de Ludwig Van Toronto (en anglais) et de Ludwig Van Montréal (en français)

\section{If You Think Classical Music is Dead, You're Dead Wrong!}

Si vous croyez que le classique est mort, nous sommes morts de rire !

Bien qu'ils soient humoristiques, ces slogans évoquent le danger imminent de mort ou de disparition, perpétuant l'image d'une forme d'art menacée qui réussit à survivre dans un contexte contemporain hostile. Si la musique classique peut être perçue comme étant en déclin par ses détracteurs, ces slogans dénotent une confiance inébranlable envers sa légitimité. Si la survie du genre implique un désir de conservation et de préservation, est-ce que cette rhétorique implique aussi un idéal de pureté et d'authenticité où l'altérité est passivement exclue et équivaut à un danger de contamination? Finalement, toute autre option médiane entre la survie et la mort, tout dénouement qui laisse place à la croissance, à l'adaptation, au compromis ou à la transformation ne fait pas partie de l'univers des possibles. C'est là que les artisans du milieu des musiques nouvelles peuvent faire des choix et s'affranchir de cette descendance eurocentrique. Ceci rappelle la vision d'une décolonialité telle qu'envisagée par Maldonado-Torres où «la production de contre-discours, de contre-savoirs, de contre-actes-créatifs et de contre-pratiques vise à démanteler la colonialité et à ouvrir la porte à de multiples autres formes d'être dans le monde» (MaldonadoTorres 2016,10$)^{16}$. La scène des musiques nouvelles peut-elle faire plus d'efforts

14 Ces critiques sont souvent énoncées sur les réseaux sociaux d'artistes qui déplorent, par exemple, l'absence de femmes dans la programmation de différents festivals de musique improvisée. En ce qui a trait aux arts médiatiques ou l'électroacoustique, je remercie Erin Gee de m'avoir dirigé vers le travail et les écrits de Tara Rodgers, Andra McCartney et Freida Abtan à ce sujet.

15 "the language and set of beliefs that characterizes Western classical music specifically as 'great' and 'universal' emerges from empire, from colonialism and capitalism ».

16 « [...] the production of counterdiscourses, counter-knowledges, counter-creative acts, and counter-practices that seek to dismantle coloniality and to open up multiple other forms of being in the world". 
pour reconnaître, repérer, inclure et célébrer ces alternatives artistiques? Les principes d'excellence, de virtuosité et de raffinement sont-ils valorisés de façon disproportionnée, au point de nous priver de plusieurs propositions artistiques riches et susceptibles de nous transformer?

\section{UNIVERSALITÉ, LÉGITIMITÉ ET MARGINALITÉ}

En 2000, Small associe déjà la notion de décentralisation au domaine musical. Stanton examine la théorie de Small qui postule que «la musique classique occidentale est une musique ethnique, comme tout autre type de musique» et pose la question à savoir "pourquoi une musique ethnique jouit-elle donc du privilège de la prétendue universalité?» (Stanton 2018,10$)^{17}$.

Stanton poursuit: «La musique classique occidentale se veut universelle parce qu'un contexte plus large de violence coloniale a facilité son ascension et la violence épistémique a facilité la naturalisation de sa primauté» (Stanton $2018,10)^{18}$.

Si la musique classique occidentale bénéficie de sa prétendue universalité et légitimité, les musiques nouvelles héritent de ce privilège aux yeux des institutions et des subventionneurs. La scène est protégée par son association à la musique classique occidentale; elle bénéficie commodément de sa légitimité automatique et présumée. Il n'est pas rare d'utiliser opportunément cette filiation historique lors d'initiatives de médiation culturelle qui visent à contextualiser et présenter le travail de compositeurs et compositrices contemporaines. En les comparant avec le travail des figures de proue de l'histoire de la musique classique, les démarches contemporaines se trouvent valorisées et bénéficient d'un transfert de légitimité. C’est un privilège gagné par les structures colonialistes.

$\mathrm{Au}$ sein de la scène des musiques nouvelles, certaines personnes espèrent une acceptation et reconnaissance plus large, d'autres préfèrent rester marginaux, axées sur la recherche et entretenant une certaine distance avec les courants dominants (musique populaire, commerciale, médiatisée). Plusieurs désirent les deux simultanément; le souhait de toucher un public plus large peut coexister avec le désir plutôt réaliste de rester en marge. Par conséquent, les membres de la scène pourraient juger leur travail comme étant pertinent, essentiel et en droit de légitimité, mais en même temps relégué à un statut de marginalité qui pourrait les exempter de penser qu'ils et elles s'inscrivent dans l'idéologie dominante, ou qu'ils et elles soient en position de pouvoir à l'égard de quiconque.

«Si la musique exprime l'identité personnelle ou collective, elle joue un rôle dans la négociation des relations entre inégaux, elle peut permettre à un groupe dominant de renforcer son hégémonie, ou à une population subordonnée de

17 «Small's theory lays bare the simple and inescapable reality that Western classical music is an ethnic music, just like any other type of music [ ... ] why does one ethnic music enjoy the privilege of so-called universality?».

18 «Western classical music fancies itself to be universal because a wider context of colonial violence facilitated its ascendency and epistemic violence facilitates the naturalization of its primacy». 
riposter à un certain degré» (Nettl 1983, 256) ${ }^{19}$. Dans ce contexte où des groupes inégaux négocient leurs relations par la musique, la scène des musiques nouvelles a le privilège d'alterner convenablement entre une position subordonnée assumée, et une position dominante inassumée. La scène a-t-elle la volonté et l'audace de renoncer à une part de ses pouvoirs pour mieux défendre les idéaux de diversité et d'inclusion? Les artistes et le public qui ne s'identifient pas à l'héritage de la musique classique démontreraient-ils un plus grand intérêt envers la scène des musiques nouvelles si cette parenté était clairement et volontairement décentralisée? La saine marginalisation du milieu, qui s'inscrit tout de même dans des idéaux artistiques et sociopolitiques, peut-elle être conservée en en élargissant sa définition, par exemple pour accueillir des approches artistiques innovatrices, mais traditionnellement non-européennes? Une transformation graduelle de la démographie ou des références culturelles de la scène des musiques nouvelles pourrait-elle mener à une plus grande légitimité et pertinence aux yeux de la société, culturellement riche et diversifiée?

\section{UNE RELATION AMBIGÜE AVEC L'APPROPRIATION CULTURELLE}

Les musiques nouvelles n'ont pas été confrontées à leur relation avec l'appropriation culturelle. En comparaison avec d'autres formes d'art où le corps, l'iconographie et les images sont impliqués, il n'y a presque pas de récriminations ou de critiques, que ce soit à l'interne ou venant de l'extérieur de la scène. Le son est-il plus abstrait que les images et que les corps? Le flux d'idées entre les traditions musicales est-il généralement plus accepté au sens éthique? Born et Hesmondhalgh ont illustré comment «le recours à d'autres musiques les musiques populaires urbaines, les musiques folkloriques et ethniques» de même que les musiques «non-artistiques » étaient considérées par les compositeurs de la modernité «comme d'autres musiques desquelles puiser de diverses manières dans leur pratique compositionnelle» (Born et Hesmondhalgh 2000, $13)^{20}$. Les cursus universitaires ont peut-être évolué depuis, mais jamais durant ma formation et mon éducation musicales de telles pratiques n'ont été abordées d'un point de vue critique.

De plus, en référence au statut marginal mais légitime de la scène, on pourrait soutenir que, s'il n'y a pas de récriminations, c'est peut-être parce que trop peu de personnes voient, entendent ou témoignent de ce que la scène produit. Certains membres de la communauté des musiques nouvelles déplorent le manque d'attention médiatique qu'elle suscite ${ }^{21}$. Je soutiens qu'en opérant dans un milieu aussi isolé de l'espace public, le milieu échappe à des critiques qui

19 «If music expresses personal or group identity, it plays a role in negotiating relationships between unequals, as a way for a dominant group to reinforce its hegemony, or for a subordinate population to fight back at some level».

20 « $[\ldots]$ the turn to other musics — urban popular musics, Western and non-Western folk and 'ethnic music' as well as 'non-art musics' were conceived by modern composers as 'others' to be drawn in a variety of ways into their compositional practice».

21 Par exemple, la rédaction du média en ligne Cette ville étrange se décrit comme une initiative qui «tente de répondre à l'absence chronique d'un espace critique véritable de la musique de création en concert dans l'espace public, et notamment dans les médias de masse dont le pouvoir discrétionnaire sur la forme et le contenu des idées véhiculées semble plus grand que jamais » (consulté le 15 
pourraient influencer nos conversations à l'interne. Le sujet de l'appropriation culturelle a provoqué des bouleversements et des controverses dans la presse et suscité des débats internationaux lorsque des créateurs et créatrices dans le domaine de la danse, du théâtre ou des arts visuels ont été critiqué pour leur engagement irresponsable envers une culture qui n'est pas la leur ${ }^{22}$. Que ces critiques soient justifiées, méritées ou pas, voilà un tout autre débat. Je soutiens par contre que ces situations provoquent une prise de conscience de problématiques contemporaines inévitables, ce qui peut être très productif pour établir un cadre décolonisateur. Ces débats montrent la coexistence d'opinions diversifiées et forcent un consensus ou des schismes parfois nécessaires.

Les membres de la scène ne se tiennent pas non plus mutuellement responsables de la dimension éthique de leurs propositions artistiques. Ce passe-droit retarde ce qui pourrait être l'occasion d'une autoréflexion utile du milieu sur sa façon d'aborder et d'utiliser les sons, les musiques et les collaborations venant de l'extérieur des limites prescrites par le genre. Comparativement aux deux dernières décennies, les aspects problématiques de l'appropriation culturelle n'étaient pas autant discutés en fin du $20^{\text {ème }}$ siècle, particulièrement dans les médias et en-dehors de la recherche académique. Bien que l'âge ne soit pas le seul facteur déterminant, j'observe des sensibilités fort différentes sur le sujet selon la génération. Si cette tension intergénérationnelle est combinée à des rapports de pouvoir entre les musiciens et musiciennes aux carrières établies et ceux et celles de la relève, il faudra encore du temps pour qu'un changement de mentalité se fasse sentir.

Ma compréhension de l'appropriation culturelle est loin d'être simpliste (Dharmoo 2019) et je ne souhaite pas faire le procès de tous les musiciens et musiciennes qui auraient emprunté de la musique ou des sons à une autre culture que la leur, que ce soit occasionnellement ou de façon régulière. Je soulève simplement le fait que les artistes de la scène des musiques nouvelles n'ont de comptes à rendre à personne - ni aux médias, ni aux chercheurs et chercheures, ni aux minorités opprimées, ni aux cultures auxquelles ils empruntent. Si un ou une artiste sonore souhaite entrer en dialogue avec d'autres musiques à travers son propre langage musical, j'encourage une réflexion approfondie sur la pertinence de cette démarche artistique, sur ce qu'elle rapporte à soi-même, versus ce qu'elle soutire des autres en termes de capital culturel, financier ou symbolique. Je tiens par contre à préciser que cet exercice autoréflexif doit comporter sa part de prises de conscience difficiles et inconfortables. Les postures de victime et de liberté d'expression brimée sont rarement productives et démontrent un manque d'écoute envers les critiques formulées.

novembre 2019 à l'adresse http://www.cettevilleetrange.org/a-propos-editos/a-propos-2013-edito-anno-iv/).

22 En novembre 2019, moment où cet article est dans sa phase finale de révision, Tanya Tagaq a dénoncé l'utilisation d'un chant katajjak dans la composition Partita de la compositrice américaine Caroline Shaw - œuvre lauréate du Prix Pulitzer en musique 2013. Étant donné l'influence médiatique et la crédibilité de Tagaq, il s'agit à ma connaissance d'une rare instance où ce regard critique «externe» mène l'artisan(e) du milieu des musiques nouvelles, dans ce cas Shaw et l'ensemble Roomful of Teeth, à offrir des excuses, des rectifications et des réparations. 


\section{L'ATTRIBUTION DU MÉRITE FONDÉE SUR LES MODES DE COMPRÉHENSION ET DE PERCEPTION DE LA CULTURE DOMINANTE}

Enriques identifie cinq étapes dans le processus de colonisation, la troisième étant le rabaissement, le dénigrement et l'insulte des systèmes et pratiques des peuples colonisés par ceux colonisateurs. Sur cette base, Laenui propose cinq phases pour la décolonisation d'un peuple, dont la première étape est la redécouverte et la récupération (Laenui 2000). À l'échelle de la scène des musiques nouvelles, il n'y a pas si longtemps, les musiques extra-européennes étaient considérées comme des curiosités exotiques, leur valeur étant à la fois idéalisée ou dépréciée. Le rejet et le dénigrement d’autres musiques par les acteurs de la scène de la musique nouvelle sont plus répandus qu'on ne souhaiterait l'admettre (ce dénigrement existe indéniablement à l'intérieur même de la scène!).

Le mérite attribué à d'autres musiques est fondé sur les façons de percevoir et de comprendre inhérentes au milieu, qui est à son tour fondé sur les façons de percevoir et de comprendre de la culture dominante. Certains types de musique seront jugés, parfois discrédités, pour leur manque de complexité, mais nous ne reconnaissons pas assez prestement que nous n'avons pas les outils nécessaires pour saisir les paramètres de difficulté et de sensibilité, ou les couches de sens inhérentes aux musiques que nous ne connaissons pas bien. L'idéalisation trop enthousiaste de ces musiques est tout aussi problématique, car ce discours se limite souvent à quelques éléments superficiels et palatables. On se convainc que l'on en comprend les subtilités, alors que nous sommes dans une forme relative d'ignorance.

Par exemple, la musique classique indienne est reconnue et applaudie pour sa complexité rythmique et son raffinement mélodique, car ce sont là des paramètres de valeur déjà présents dans la musique classique occidentale. Il s'agit aussi d'une musique "classique» pratiquée et appréciée par l'élite indienne, donc jugée comme étant sophistiquée et raffinée. À l'opposé, le hip-hop est encore fortement dénigré malgré ses mérites, mesurés selon d'autres paramètres et d'autres échelles de valeur. Selon Cervantes, «l'art du rap ne se limite pas à faire rimer les mots; la pratique implique le contrôle de la respiration, le sens de l'organisation, le rythme, la capacité d'articuler sa voix et de transmettre des messages qui reflètent l'expérience et la condition sociale» (Cervantes 2015, $8)^{23}$.

Entre le rejet et l'idéalisation, il y a évidemment d'autres façons d'apprécier les musiques qui ne nous sont pas familières - les artistes devraient pouvoir nourrir leur inspiration sans sélectivement les filtrer à la source - mais ceci peut se faire avec plus de curiosité et de modestie. L'ignorance à l'égard de telle ou telle musique ne doit pas nécessairement être source de honte, ou déguisée

23 « [... ] rapping was more than making words simply rhyme and that the practice involves breath control, organization skills, rhythm, the ability to articulate your voice, and conveying messages that reflect social experience and condition ". 
par un pseudo-intellectualisme. L'ignorance peut être assumée et provoquer un réel désir d'éducation.

La critique que Stanton fait à propos de l'universalité de la musique classique occidentale et «sa propension subséquente à délégitimer, à subordonner, à s'approprier et à tokéniser d'autres systèmes de valeur esthétique» peut raisonnablement être appliquée à la scène de la musique nouvelle (Stanton 2018, $10)^{24}$.

Inversons le scénario. Pour les musiciens qui n'appartiennent pas à la scène, la musique nouvelle est sans doute critiquée pour son manque de rythme, l'absence d'une mélodie reconnaissable, le fait qu'elle soit si cérébrale, le fait qu'on ne puisse danser ou bouger dessus, le fait qu'on doive rester assis en silence pour l'écouter ... Selon mon expérience, une grande part des membres de la scène des musiques nouvelles ne sent pas le besoin de se défendre contre de telles critiques. Elle reste confiante et inébranlable dans la légitimité de la scène, ou encore elle se complait à son statut de marginalité, acceptant sans scrupule son inintelligibilité pour quiconque n'en détient pas les codes. Les critiques provenant d'autres façons de sentir ou de comprendre la musique, qui peuvent par ailleurs être formulées tant par des néophytes que par des sommités musicales d'une autre tradition, sont automatiquement rejetées. Ces critiques ne sont pas envisagées comme des opportunités de croissance qui pourraient transcender les limites du genre. Si la musique nouvelle est obsédée par son propre progrès, ce progrès est accompli selon ses propres termes. Évidemment, plusieurs musiciens ou musiciennes, et compositeurs ou compositrices, sont à l'écoute des critiques formulées ci-haut, mais plus les pratiques artistiques vont dans cette direction (renouer avec le rythme, la mélodie, la corporalité) plus elles ont tendance à se dissocier de la scène des musiques nouvelles, ou encore, ces approches deviennent jugées et rejetées à l'interne.

\section{VERS UN ART DÉCOLONIAL: QUELQUES PISTES DE SOLUTION POUR LA SCÈNE DES MUSIQUES NOUVELLES}

Je reconnais que plusieurs individus et organismes en sont déjà à l'étape de regarder vers l'avant, d'adopter des mesures, de produire des œuvres et de contribuer au processus de transformation de la scène. Les recommandations pour la Society for American Music (Levitz 2017) et les rapports de consultation de Diversité Artistique Montréal (Hajji 2018) et de Orchestres Canada (Attariwala, Peerbaye 2019) sont de bons outils pour quiconque souhaite améliorer ses actions et sa compréhension de la décolonisation des arts, bien qu'ils n'aient pas été conçus sur mesure pour les acteurs de la scène des musiques nouvelles.

Quant aux créateurs, artiste sonores et compositeurs autochtones ou appartenant à la diversité culturelle (et je m'inclus à ce groupe), nous pourrions davantage penser à la décolonisation par l'art.

24 «[Classical music's] universality and subsequent propensity to delegitimize, subordinate, appropriate, and tokenize other systems of aesthetic value». 
Nous sommes entrés dans une phase nouvelle : celle de la décolonisation par l'art. Le processus de décolonisation de l'art était fondé sur la résilience, la résistance, la contestation et la critique. Le processus de décolonisation par l'art, quant à lui, vise à compléter la réinscription des peuples autochtones dans l'histoire politique, en utilisant l'art comme avant-garde. Ce processus est loin d'être arrivé à son terme (Sioui Durand 2018, 24).

En tant qu'artistes individuels, comment notre travail peut-il rompre avec ou aller au-delà des modèles standardisés de créer, de penser et de sentir la musique et les arts sonores? Notre travail artistique pourrait remettre en question les cadres normatifs hérités des modèles eurocentriques, cadres qui ont été perpétués par le racisme systémique institutionnalisé. Nos œuvres pourraient être présentées et vécues dans différents espaces, touchant un plus grand nombre de publics et de personnes. Notre travail peut aller bien au-delà de la création musicale et avoir une dimension sociale qui pourrait inclure le mentorat, l'éducation, la médiation culturelle et la prise de parole en public. Le maintien d'une pratique artistique individuelle multidirectionnelle contribue à développer un sens d'adaptation et de négociation constante, évitant le dogmatisme, les discours dominants ou l'hégémonie. En repensant nos rôles, nous pourrions placer l'engagement et la pertinence sociale au cour de nos préoccupations. De plus, nous pourrions diminuer l'importance du prestige accordé au contexte de présentation de notre travail artistique. L'œuvre peut vivre à l'intérieur et à l'extérieur des institutions dominantes, avec ou sans l'approbation de directions artistiques renommées ou de pairs respectés. La pertinence sociale de nos œuvres est susceptible d'augmenter par son contact avec une plus grande variété d'individus.

La musique décoloniale se manifeste souvent en résistance active contre les institutions, dans une relation externe. C'est à partir de l'extériorité que les méthodes de lutte décoloniales peuvent imprégner et transformer radicalement la fonction hégémonique des institutions dominantes (Stanton 2018,11$)^{25}$.

Je réfléchis personnellement à ces questions par rapport à ma propre pratique artistique et à ma façon de naviguer le contexte professionnel et social de la scène des musiques nouvelles. Plusieurs autres artistes y réfléchissent également. Collectivement, nous avons le potentiel d'inventer une multitude de nouvelles expressions artistiques et de remettre en question les façons dominantes de vivre l'expérience musicale - un bel écho à la décolonialité selon Maldonado-Torres qui vise la production d'une multiplicité de contre-discours et de contre-pratiques (Maldonado-Torres 2016, 10).

La responsabilité du changement existe à différentes échelles. Elle appartient aux organismes culturels subventionnés qui sont soutenus pour réaliser leurs mandats envers la culture à l'échelle municipale, provinciale, nationale

25 «Decolonial musicking often exists in active resistance against institutions, in an exterior relation. It is from exteriority that decolonial methods of struggle can permeate and radically transform the hegemonic function of dominant institutions ». 
et internationale. Elle appartient aux organismes, qui se doivent de rechercher, de commander, d'organiser et de programmer ces nouvelles formes d'art novatrices, socialement pertinentes et décoloniales. Mais les artistes doivent avoir une longueur d'avance, car ce processus de décolonisation commence par l'art, notre art.

\section{Conclusion}

La scène des musique nouvelles a la volonté affichée de projeter une image de progressivité et d'innovation. Une certaine part de ses membres tient à comprendre comment elle peut devenir encore plus inclusive, équitable et représentative du tissu social contemporain - affranchie de la colonialité du pouvoir. Cependant, le partage de ces valeurs n'est pas unanime. Alors que certains membres de la communauté initient ou contribuent à son processus de décolonisation, d'autres l'ignorent, le méconnaissent, y résistent ou bien y contreviennent.

Une grande part des membres de la scène des musiques nouvelles n'accepte pas ou ne reconnaît qu'avec beaucoup de difficultés de quelles manières le racisme systémique et les obstacles socioéconomiques entrent en jeu dans les trajectoires de ses artisans et de celles et ceux qui en demeurent exclus, ou méconnus. Les personnes en situation de pouvoir dans la scène des musiques nouvelles se cachent souvent derrière le fait qu'elles n'ont aucune mauvaise intention. On entend des excuses qui seraient inacceptables, ou du moins très peu crédibles, dans d'autres disciplines comme le théâtre et la danse. Il n'est pas rare d'entendre, par exemple, l'argument de l'invisibilité ( Je ne pense pas même qu'il y ait de compositrices noires, comment peut-on les programmer dans notre série de concerts?»), d'indifférence à la couleur ("Je ne vois pas la couleur de peau quand j'écoute une œuvre, c'est la musique qui compte!») ou encore l'argument de la qualité («Nous n’avons pas pu trouver une chanteuse asiatique assez qualifiée pour jouer ce rôle.») Si une personne en situation de pouvoir s'enorgueillit de sa vision généralement progressiste sur les questions sociales, ces excuses dénotent une réticence à reconnaître ses propres préjugés. Pour l'ensemble de la scène, ces excuses, combinées à une attitude généralement progressiste, confortent un sentiment d'innocence et de distanciation, une posture typique dans les contextes systémiques de colonialité.

Ne pas reconnaitre comment les inégalités systémiques affectent la scène de la musique nouvelle est un déni malsain qui maintient le statu quo. La présomption qu'on ne puisse rien faire ou rien changer maintient le statu quo. Dans l'avant-propos du Rapport de la consultation sur le racisme systémique dans le milieu des arts, de la culture et des médias à Montréal commandé par Diversité Artistique Montréal (Hajji 2018), le directeur général Jérôme Pruneau rappelle aux lecteurs et lectrices que:

[il] ne s'agit donc pas ici de pointer des individus du doigt, mais bien d'accepter l'idée que des mécaniques administratives et organisationnelles, bien qu'opérées par des individus pas forcément racistes, engendrent des situations inégalitaires et oppressantes (Hajji 2018, 8). 
Il en demeure que ce sont des individus qui prennent des décisions et qui mettent en œuvre différentes initiatives, ce sont des individus qui créent et produisent des œuvres artistiques.

Évidemment, les reflets de la colonialité ne sont pas spécifiques à la musique nouvelle, ils se manifestent dans plusieurs communautés, à plusieurs échelles. Cependant, le processus de transformation qui pourra mener à un avenir décolonisé doit commencer par la reconnaissance de multiples façons dont la colonialité se reflète à l'échelle des milieux auxquels nous appartenons, des milieux qui nous appartiennent.

\section{RÉFÉRENCES}

Attariwala, Parmela. 2019. Correspondance personnelle avec l'auteur. Reproduite avec permission.

Attariwala, Parmela et Soraya Peerbaye. 2019. «L'orchestre retentit: les relations entre les orchestres canadiens, les peuples autochtones et les personnes de couleur». Rapport de consultation commandé par Orchestres Canada. Consulté à l'adresse : https://oc.ca/fr/resource/lorchestre-retentit/

Bhagwati, Sandeep. 2018. «Glossaire raisonné». Circuit - Musiques contemporaines 28 (1): 15-22.

Born, Georgina et David Hesmondhagh (éds.). 2000. Western music and its others: Difference, Representation, and Appropriation in Music. Berkeley: University of California Press.

Cervantes, Marco Antonio. 2015. «Teaching Decolonial Sounds on the Margins: Reflections on a K-12 Teacher Workshop Covering Black \& Brown Musical Transculturation in Texas». Multicultural Education 22 (3/4): 8-14.

Couture, Ariane. 2019. «Jouer les femmes: les concerts de musique contemporaine au Québec». Recherches féministes 32 (1): 195-215.

Dharmoo, Gabriel. 2019. "Anthropologies imaginaires: Une critique de la colonialité par la voix et la satire». Anthropologie et Sociétés 43 (1): 141-168.

Green, Anthony R. 2018. "What the Optics of New Music Say to Black Composers ». Site internet New Music USA. Consulté à l'adresse: https://nmbx. newmusicusa.org/what-the-optics-of-new-music-say-to-black-composers/

Hajji, Nadia. 2018. Pour un processus d'équité culturelle: Rapport de la consultation sur le racisme systémique dans le milieu des arts, de la culture et des médias à Montréal. Montréal : Diversité Artistique Montréal (DAM).

Laenui, Poka (Burgess, Hayden F.) 2000. «Processes of Decolonization». Dans Reclaiming Indigenous Voice and Vision, sous la dir. de Marie Battiste, 150160. Vancouver: University of British Columbia Press.

Levitz, Tamara. 2017. "Decolonizing the Society for American Music ». The Bulletin of the Society for American Music 43 (3) : 1-13.

Maldonado-Torres, Nelson. 2016. «Outline of Ten Theses on Coloniality and Decoloniality ». Site internet Frantz Fanon Foundation. Consulté à l'adresse: https://fondation-frantzfanon.com/wp-content/uploads/2018/10/maldonado-torres_outline_of_ten_theses-10.23.16.pdf 
Nettl, Bruno. 1983. The Study of Ethnomusicology: thirty-one issues and concepts. Urbana: University of Illinois Press.

Robinson, Dylan. 2012. "Intercultural Art Music and the Sensory Veracity of Reconciliation: Brent Michael Davids' Powwow Symphony on the Dakota Music Tour» MUSICultures 39 (1). Consulté à l'adresse : https://journals. lib.unb.ca/index.php/MC/article/view/19997

Sioui Durand, Guy. 2018. "Autochtones: de la décolonisation de l'art par l'art ». Liberté-Art \& Politique (321) : 24-26.

Stanton, Burke. 2018. "Musicking in the Borders: Toward Decolonizing Methodologies». Philosophy of Music Education Review 26 (1) : 4-23.

Tuck, Eva et K. Wayne Yang. 2012. «Decolonization is not a metaphor »Decolonization: Indigeneity, Education \& Society 1 (1) : 1-40.

Yoshihara, Mari. 2007. Musicians from a Different Shore: Asians and Asian Americans in Classical Music. Philadelphia : Temple University Press.

\title{
RÉSUMÉ
}

Certains membres de la scène des musiques nouvelles souhaitent en décentrer ses racines eurocentriques et en critiquer ses tendances colonialistes. Avant même de discuter des stratégies qui pourraient constituer un cadre décolonisateur, il est utile d'identifier comment la colonialité se reflète dans cette scène. L'auteur, lui-même membre actif de celle-ci, partage des pistes de réflexion portant sur l'homogénéité culturelle du milieu, les questions d'accès, l'héritage de la musique classique, le concept de l'excellence européenne, la présomption d'universalité, la coexistence de statuts de légitimité et de marginalité, la relation ambigüe avec l'appropriation culturelle et les fondements de l'attribution du mérite.

\begin{abstract}
Some members of the new music scene wish to decentralize its Eurocentric roots and criticize its colonialist tendencies. Prior to the discussion of strategies that could constitute a decolonizing framework, it is useful to identify how coloniality is reflected in this scene. The author, himself an active member of this scene, shares avenues of reflection on the cultural homogeneity of the milieu, questions of access, the legacy of classical music, the concept of European excellence, the presumption of universality, the coexistence of legitimacy and marginality, the ambiguous relationship with cultural appropriation and the foundations of the attribution of merit.
\end{abstract}

\section{BIOGRAPHIE}

Gabriel Dharmoo est compositeur, vocaliste, improvisateur et chercheur actif à l'international. Il est lauréat des Prix Jules-Léger (2017) et Robert-Fleming (2011) du Conseil des Arts du Canada, du Concours de composition MusCan (2017), du Prix d'Europe de composition Fernand-Lindsay (2011), et de différents prix de la SOCAN. Il est candidat au doctorat en études interdisciplinaires à Concordia, travaillant avec Sandeep Bhagwati, Noah Drew et David Howes. 